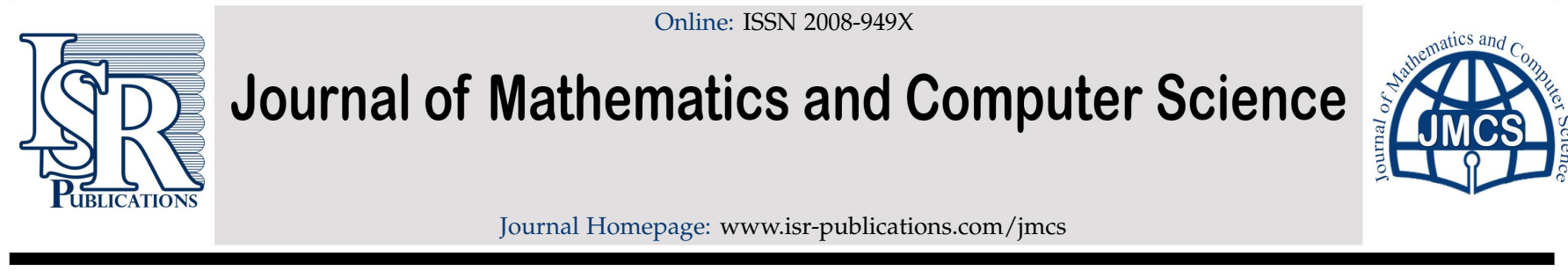

\title{
Fisher type fixed point results in controlled metric spaces
}

\section{Durdana Lateef}

Department of Mathematics, College of Science, Taibah University, Madina, 41411, Saudi Arabia.

\begin{abstract}
In the present paper, we define a rational contractive condition of Fisher type in the context of controlled metric space and obtain some generalized fixed point results in this space. These results will unify and amend many well-known results of literature. Some consequences and an example has been presented at the end to show the authenticity of the established results.
\end{abstract}

Keywords: Fixed point, rational contraction, controlled metric spaces.

2010 MSC: 47H10, 54H25, 65Q10.

(C)2020 All rights reserved.

\section{Introduction}

In 1906, the self-evident progress of a metric space was basically given by Frechet. Banach's fixed point theorem (BFPT) [9] is one of the crucial problems of fixed point theory and its utility, which states that, if $\mathcal{V}:(\mathcal{Q}, \varphi) \rightarrow(\mathcal{Q}, \varphi)$ (complete metric space) and $\exists \alpha \in[0,1)$ such that

$$
\varphi\left(\mathcal{V}_{\rho}, \mathcal{V}_{\kappa}\right) \leqslant \alpha \varphi(\rho, \kappa), \quad \forall \rho, \kappa \in \mathcal{Q},
$$

then $\exists \rho^{*} \in \mathcal{Q}$ such that $\mathcal{V} \rho^{*}=\rho^{*}$. It is evident from the above contractive condition that $\mathcal{V}$ is a continuous function. Due to importance and simplicity of BFPT, many authors (see [1-23] and references therein) have obtained lots of fascinating upgrade and adjunct of it.

In 1980 Fisher [11] established a result for mapping satisfying

$$
\varphi\left(\mathcal{V} \rho, \mathcal{V}_{\kappa}\right) \leqslant \alpha \varphi(\rho, \kappa)+\beta \frac{\varphi(\rho, \mathcal{V} \rho) \varphi\left(\kappa, \mathcal{V}_{\kappa}\right)}{1+\varphi(\rho, \kappa)}, \quad \forall \rho, \kappa \in \mathcal{Q},
$$

where $\alpha, \beta \in[0,1)$. Several researchers followed Fisher [11] paper using different types of contractive conditions in metric spaces.

Inspired from this innovative idea, several mathematicians generalized and extended this conception in the recent years as: semi metric spaces, rectangular metric spaces, quasi-semimetric spaces, quasi metric spaces, probabilistic metric spaces, pseudo metric spaces, extended b-metric space (EbMs), partial metric spaces, b-metric space (bMS) and controlled metric space (C-MS), etc.

Czerwik [10] defined the notion of (bMS) as follows:

Email address: drdurdanamaths@gmail.com (Durdana Lateef)

doi: $10.22436 /$ jmcs.020.03.06

Received: 2019-09-14 Revised: 2019-11-11 Accepted: 2019-11-27 
Definition 1.1 (see [10]). Let $Q \neq \emptyset$ and $s \geqslant 1$ and $\varphi: Q \times Q \rightarrow[0, \infty)$. If

(b1) $\varphi(\rho, \kappa)=0 \Leftrightarrow \rho=\kappa ;$

(b2) $\varphi(\rho, \kappa)=\varphi(\kappa, \rho), \forall \rho, k \in Q$;

(b3) $\varphi(\rho, \omega) \leqslant s[\varphi(\rho, \kappa)+\varphi(\kappa, \omega)], \forall \rho, \kappa, \omega \in \mathcal{Q}$,

then $(Q, \varphi)$ is called a b-MS.

In 2017, Kamran et al. [16] initiated the concept of (EbMS).

Definition 1.2. Let $\mathcal{Q} \neq \emptyset$ and $\sigma: \mathcal{Q} \times \mathcal{Q} \rightarrow[1, \infty)$ and $\varphi: \mathcal{Q} \times \mathcal{Q} \rightarrow[0, \infty)$. If

(i) $\varphi(\rho, \kappa)=0 \Leftrightarrow \rho=\kappa$;

(ii) $\varphi(\rho, \kappa)=\varphi(\kappa, \rho)$;

(iii) $\varphi(\rho, \kappa) \leqslant \sigma(\rho, \kappa)[\varphi(\rho, \omega)+\varphi(\kappa, \omega)]$,

then $(Q, \varphi)$ is called an EbMS.

Recently, a new kind of a generalized bMS introduced by Mlaiki et al. [21].

Definition 1.3 ([21]). Let $\mathcal{Q} \neq \emptyset$ and $\sigma: \mathcal{Q} \times \mathcal{Q} \rightarrow[1, \infty)$ and $\varphi: \mathcal{Q} \times \mathcal{Q} \rightarrow[0, \infty)$. If

(i) $\varphi(\rho, \kappa)=0 \Leftrightarrow \rho=\kappa$;

(ii) $\varphi(\rho, \kappa)=\varphi(\kappa, \rho)$;

(iii) $\varphi(\rho, \kappa) \leqslant \sigma(\rho, \omega) \varphi(\rho, \omega)+\sigma(\kappa, \omega) \varphi(\kappa, \omega)$,

then $(Q, \varphi, \sigma)$ is called a (C-MS).

Definition 1.4 ([21]). Let $\left\{\rho_{r}\right\}_{r} \geqslant 0$ be a sequence in $(Q, \varphi, \sigma)$.

(1) $\left\{\rho_{r}\right\}_{r} \geqslant 0 \rightarrow \rho$ in $Q$, is convergent if $\forall \epsilon>0, \exists N=N(\epsilon) \in \mathbb{N}$ such that $\varphi\left(\rho_{r}, \rho\right)<\epsilon, \forall r \geqslant N$.

(2) $\left\{\rho_{r}\right\}_{r} \geqslant 0$ is Cauchy, if $\forall \epsilon>0, \exists N=N(\epsilon) \in \mathbb{N}$ such that $\varphi\left(\rho_{m}, \rho_{r}\right)<\epsilon, \forall m, r \geqslant N$.

(3) If every Cauchy sequence in $(Q, \varphi, \sigma)$ is convergent then $(Q, \varphi, \sigma)$ is complete.

Theorem 1.5 ([21]). Let $(Q, \varphi, \sigma)$ be a complete C-MS. Let $\mathcal{V}: Q \rightarrow Q$ be such that

$$
\varphi\left(\mathcal{V}_{\rho}, \mathcal{V}_{\kappa}\right) \leqslant \alpha(\varphi(\rho, \kappa)), \quad \forall \rho, \kappa \in \mathcal{Q},
$$

where $\alpha \in[0,1)$. For $\rho_{0} \in \mathcal{Q}$, take $\rho_{\mathrm{r}}=\mathcal{V}^{\mathrm{r}} \rho_{0}$. Suppose that

$$
\sup _{m \geqslant 1} \lim _{i \rightarrow \infty} \frac{\sigma\left(\rho_{i+1}, \rho_{i+2}\right) \sigma\left(\rho_{i+1}, \rho_{m}\right)}{\sigma\left(\rho_{i}, \rho_{i+1}\right)}<\frac{1}{\lambda} .
$$

Assume that $\forall \rho \in \mathcal{Q}, \lim _{\mathrm{r} \rightarrow \infty} \sigma\left(\rho_{\mathrm{r}}, \rho\right)$ and $\lim _{\mathrm{r} \rightarrow \infty} \sigma\left(\rho, \rho_{\mathrm{r}}\right)$ exist and are finite. Then, $v$ has a unique fixed point.

In this paper, we define rational contraction of Fisher [11] type in the context of C-MS and prove some new fixed point result. Also, we present a non trivial example to illustrate importance of proved results. 


\section{Main results}

Theorem 2.1. Let $(Q, \varphi, \sigma)$ be a complete C-MS. Let $\mathcal{V}: \mathcal{Q} \rightarrow Q$ be such that

$$
\varphi\left(\mathcal{V} \rho, \mathcal{V}_{\kappa}\right) \leqslant \alpha \varphi(\rho, \kappa)+\beta \frac{\varphi(\rho, \mathcal{V} \rho) \varphi\left(\kappa, \mathcal{V}_{\kappa}\right)}{1+\varphi(\rho, \kappa)}
$$

$\forall \rho, \kappa \in Q$, where $\alpha, \beta \in[0,1)$ such that $\lambda=\alpha+\beta<1$. For $\rho_{0} \in \mathcal{Q}$, take $\rho_{\mathrm{r}}=\mathcal{V}^{\mathrm{r}} \rho_{0}$. Suppose that

$$
\sup _{m \geqslant 1} \lim _{i \rightarrow \infty} \frac{\sigma\left(\rho_{i+1}, \rho_{i+2}\right) \sigma\left(\rho_{i+1}, \rho_{m}\right)}{\sigma\left(\rho_{i}, \rho_{i+1}\right)}<\frac{1}{\lambda}
$$

Next, assume that $\forall \rho \in Q$, we have $\lim _{\mathrm{r} \rightarrow \infty} \sigma\left(\rho_{\mathrm{r}}, \rho\right)$ and $\lim _{\mathrm{r} \rightarrow \infty} \sigma\left(\rho, \rho_{\mathrm{r}}\right)$ exist and are finite. Then, $v$ has a unique fixed point.

Proof. Let $\rho_{0} \in \mathcal{Q}$. We construct $\left\{\rho_{\mathrm{r}}\right\}$ in $\mathcal{Q}$ by $\rho_{\mathrm{r}+1}=\mathcal{V} \rho_{\mathrm{r}}, \forall \mathrm{r} \in \mathbb{N}$. If $\exists \mathrm{r}_{0} \in \mathbb{N}$ for which $\rho_{\mathrm{r}_{0}+1}=\rho_{\mathrm{r}_{0}}$, then $\mathcal{\nu} \rho_{r_{0}}=\rho_{r_{0}}$. Hence the proof is finished. Now, we assume that $\rho_{\mathrm{r}+1} \neq \rho_{\mathrm{r}} \forall \mathrm{r} \in \mathbb{N}$. Thus by (2.1), we get

$$
\begin{aligned}
\varphi\left(\rho_{r}, \rho_{r+1}\right) & =\varphi\left(\mathcal{V} \rho_{r-1}, \mathcal{V} \rho_{r}\right) \\
& \leqslant \alpha \varphi\left(\rho_{r-1}, \rho_{r}\right)+\beta \frac{\varphi\left(\rho_{r-1}, \mathcal{V} \rho_{r-1}\right) \varphi\left(\rho_{r}, \mathcal{V} \rho_{r}\right)}{1+\varphi\left(\rho_{r-1}, \rho_{r}\right)} \\
& =\alpha \varphi\left(\rho_{r-1}, \rho_{r}\right)+\beta \frac{\varphi\left(\rho_{r-1}, \rho_{r}\right) \varphi\left(\rho_{r}, \rho_{r+1}\right)}{1+\varphi\left(\rho_{r-1}, \rho_{r}\right)} \\
& \leqslant \alpha \varphi\left(\rho_{r-1}, \rho_{r}\right)+\beta \varphi\left(\rho_{r}, \rho_{r+1}\right),
\end{aligned}
$$

which implies

$$
\varphi\left(\rho_{r}, \rho_{r+1}\right) \leqslant\left(\frac{\alpha}{1-\beta}\right) \varphi\left(\rho_{r-1}, \rho_{r}\right)=\lambda \varphi\left(\rho_{r-1}, \rho_{r}\right) .
$$

Similarly,

$$
\begin{aligned}
& \varphi\left(\rho_{\mathrm{r}-1}, \rho_{\mathrm{r}}\right)=\varphi\left(\mathcal{V} \rho_{\mathrm{r}-2}, \mathcal{V} \rho_{\mathrm{r}-1}\right) \\
& \leqslant \alpha \varphi\left(\rho_{r-2}, \rho_{r-1}\right)+\beta \frac{\varphi\left(\rho_{r-2}, \mathcal{V} \rho_{r-2}\right) \varphi\left(\rho_{r-1}, \mathcal{V} \rho_{r-1}\right)}{1+\varphi\left(\rho_{r-2}, \rho_{r-1}\right)} \\
& =\alpha \varphi\left(\rho_{r-2}, \rho_{r-1}\right)+\beta \frac{\varphi\left(\rho_{r-2}, \rho_{r-1}\right) \varphi\left(\rho_{r-1}, \rho_{r}\right)}{1+\varphi\left(\rho_{r-2}, \rho_{r-1}\right)} \\
& =\alpha \varphi\left(\rho_{\mathrm{r}-2}, \rho_{\mathrm{r}-1}\right)+\beta \varphi\left(\rho_{\mathrm{r}-1}, \rho_{\mathrm{r}}\right) \text {, }
\end{aligned}
$$

which implies that

$$
\varphi\left(\rho_{r-1}, \rho_{r}\right) \leqslant\left(\frac{\alpha}{1-\beta}\right) \varphi\left(\rho_{r-2}, \rho_{r-1}\right)=\lambda \varphi\left(\rho_{r-2}, \rho_{r-1}\right) .
$$

Pursuing in this direction, we get

$$
\begin{aligned}
\varphi\left(\rho_{r}, \rho_{r+1}\right) & \leqslant \lambda \varphi\left(\rho_{r-1}, \rho_{r}\right) \\
& \leqslant \lambda^{2} \varphi\left(\rho_{r-2}, \rho_{r-1}\right) \\
& \leqslant \\
& \vdots \\
& \leqslant \lambda^{r} \varphi\left(\rho_{0}, \rho_{1}\right) .
\end{aligned}
$$

Thus,

$$
\varphi\left(\rho_{r}, \rho_{r+1}\right) \leqslant \lambda^{r} \varphi\left(\rho_{0}, \rho_{1}\right)
$$


For all $r, m \in \mathbb{N}(r<m)$, we have

$$
\begin{aligned}
\varphi\left(\rho_{r}, \rho_{m}\right) \leqslant & \sigma\left(\rho_{r}, \rho_{r+1}\right) \varphi\left(\rho_{r}, \rho_{r+1}\right)+\sigma\left(\rho_{r+1}, \rho_{m}\right) \varphi\left(\rho_{r+1}, \rho_{m}\right) \\
\leqslant & \sigma\left(\rho_{r}, \rho_{r+1}\right) \varphi\left(\rho_{r}, \rho_{r+1}\right)+\sigma\left(\rho_{r+1}, \rho_{m}\right) \sigma\left(\rho_{r+1}, \rho_{r+2}\right) \varphi\left(\rho_{r+1}, \rho_{r+2}\right) \\
& +\sigma\left(\rho_{r+1}, \rho_{m}\right) \sigma\left(\rho_{r+2}, \rho_{m}\right) \varphi\left(\rho_{r+2}, \rho_{m}\right) \\
\leqslant & \sigma\left(\rho_{r}, \rho_{r+1}\right) \varphi\left(\rho_{r}, \rho_{r+1}\right)+\sigma\left(\rho_{r+1}, \rho_{m}\right) \sigma\left(\rho_{r+1}, \rho_{r+2}\right) \varphi\left(\rho_{r+1}, \rho_{r+2}\right) \\
& +\sigma\left(\rho_{r+1}, \rho_{m}\right) \sigma\left(\rho_{r+2}, \rho_{m}\right) \sigma\left(\rho_{r+2}, \rho_{r+3}\right) \varphi\left(\rho_{r+2}, \rho_{r+3}\right) \\
& +\sigma\left(\rho_{r+1}, \rho_{m}\right) \sigma\left(\rho_{r+2}, \rho_{m}\right) \sigma\left(\rho_{r+3}, \rho_{m}\right) \varphi\left(\rho_{r+3}, \rho_{m}\right) \\
\leqslant & \\
\vdots & \quad \sum_{i=r+1}^{m-2}\left(\prod_{j=r+1}^{i} \sigma\left(\rho_{j}, \rho_{m}\right)\right) \sigma\left(\rho_{i}, \rho_{i+1}\right) \varphi\left(\rho_{i}, \rho_{i+1}\right) \\
\leqslant & \sigma\left(\rho_{r}, \rho_{r+1}\right) \varphi\left(\rho_{r}, \rho_{r+1}\right)+\sum_{i=r+1}^{m-1} \sigma\left(\rho_{i}, \rho_{m}\right) \varphi\left(\rho_{m-1}, \rho_{m}\right),
\end{aligned}
$$

which further implies that

$$
\begin{aligned}
\varphi\left(\rho_{r}, \rho_{m}\right) \leqslant & \sigma\left(\rho_{r}, \rho_{r+1}\right) \varphi\left(\rho_{r}, \rho_{r+1}\right)+\sum_{i=r+1}^{m-2}\left(\prod_{j=r+1}^{i} \sigma\left(\rho_{j}, \rho_{m}\right)\right) \sigma\left(\rho_{i}, \rho_{i+1}\right) \varphi\left(\rho_{i}, \rho_{i+1}\right) \\
& +\left(\prod_{i=r+1}^{m-1} \sigma\left(\rho_{i}, \rho_{m}\right)\right) \sigma\left(\rho_{m-1}, \rho_{m}\right) \varphi\left(\rho_{m-1}, \rho_{m}\right) \\
\leqslant & \sigma\left(\rho_{r}, \rho_{r+1}\right) \lambda^{r} \varphi\left(\rho_{0}, \rho_{1}\right)+\sum_{i=r+1}^{m-2}\left(\prod_{j=r+1}^{i} \sigma\left(\rho_{j}, \rho_{m}\right)\right) \sigma\left(\rho_{i}, \rho_{i+1}\right) \lambda^{i} \varphi\left(\rho_{0}, \rho_{1}\right) \\
& +\left(\prod_{i=r+1}^{m-1} \sigma\left(\rho_{i}, \rho_{m}\right)\right) \sigma\left(\rho_{m-1}, \rho_{m}\right) \lambda^{m-1} \varphi\left(\rho_{0}, \rho_{1}\right) \\
= & \sigma\left(\rho_{r}, \rho_{r+1}\right) \lambda^{r} \varphi\left(\rho_{0}, \rho_{1}\right)+\sum_{i=r+1}^{m-1}\left(\prod_{j=r+1}^{i} \sigma\left(\rho_{j}, \rho_{m}\right)\right) \sigma\left(\rho_{i}, \rho_{i+1}\right) \lambda^{i} \varphi\left(\rho_{0}, \rho_{1}\right) .
\end{aligned}
$$

Thus

$$
\varphi\left(\rho_{r}, \rho_{m}\right) \leqslant \sigma\left(\rho_{r}, \rho_{r+1}\right) \lambda^{r} \varphi\left(\rho_{0}, \rho_{1}\right)+\sum_{i=r+1}^{m-1}\left(\prod_{j=r+1}^{i} \sigma\left(\rho_{j}, \rho_{m}\right)\right) \sigma\left(\rho_{i}, \rho_{i+1}\right) \lambda^{i} \varphi\left(\rho_{0}, \rho_{1}\right) .
$$

Let

$$
S_{l}=\sum_{i=0}^{l}\left(\prod_{j=0}^{i} \sigma\left(\rho_{j}, \rho_{m}\right)\right) \sigma\left(\rho_{i}, \rho_{i+1}\right) \lambda^{i} \varphi\left(\rho_{0}, \rho_{1}\right) \text {. }
$$

From (2.2), we get

$$
\varphi\left(\rho_{r}, \rho_{m}\right) \leqslant \varphi\left(\rho_{0}, \rho_{1}\right)\left[\lambda^{r} \sigma\left(\rho_{r}, \rho_{r+1}\right)+\left(S_{m-1}-S_{r}\right)\right] .
$$

As above, using $\sigma(\rho, \kappa) \geqslant 1$, and ratio test, $\lim _{r \rightarrow \infty} S_{r}$ exists. Thus $\left\{S_{r}\right\}$ is Cauchy. Finally, letting $r, m \rightarrow \infty$ in (2.3), we conclude that

$$
\lim _{r, m \rightarrow \infty} \varphi\left(\rho_{r}, \rho_{m}\right)=0
$$


Thus, $\left\{\rho_{\mathrm{r}}\right\}$ is a Cauchy $(\mathcal{Q}, \varphi, \sigma)$. So $\exists \rho^{*} \in \mathcal{Q}$ such that

$$
\lim _{r \rightarrow \infty} \varphi\left(\rho_{r}, \rho^{*}\right)=0,
$$

that is, $\rho_{r} \rightarrow \rho^{*}$ as $r \rightarrow \infty$. Now, by (2.1) and condition (iii), we get

$$
\begin{aligned}
\varphi\left(\rho^{*}, \mathcal{V} \rho^{*}\right) & \leqslant \sigma\left(\rho^{*}, \rho_{r+1}\right) \varphi\left(\rho^{*}, \rho_{r+1}\right)+\sigma\left(\rho_{r+1}, \mathcal{V} \rho^{*}\right) \varphi\left(\rho_{r+1}, \mathcal{V} \rho^{*}\right) \\
& =\sigma\left(\rho^{*}, \rho_{r+1}\right) \varphi\left(\rho^{*}, \rho_{r+1}\right)+\sigma\left(\rho_{r+1}, \mathcal{V} \rho^{*}\right) \varphi\left(\mathcal{V} \rho_{r}, \mathcal{V} \rho^{*}\right) \\
& \leqslant \sigma\left(\rho^{*}, \rho_{r+1}\right) \varphi\left(\rho^{*}, \rho_{r+1}\right)+\sigma\left(\rho_{r+1}, \mathcal{V} \rho^{*}\right)\left[\alpha \varphi\left(\rho_{r}, \rho^{*}\right)+\beta \frac{\varphi\left(\rho_{r}, \mathcal{V} \rho_{r}\right) \varphi\left(\rho^{*}, \mathcal{V} \rho^{*}\right)}{1+\varphi\left(\rho_{r}, \rho^{*}\right)}\right] \\
& =\sigma\left(\rho^{*}, \rho_{r+1}\right) \varphi\left(\rho^{*}, \rho_{r+1}\right)+\sigma\left(\rho_{r+1}, \mathcal{V} \rho^{*}\right)\left[\alpha \varphi\left(\rho_{r}, \rho^{*}\right)+\beta \frac{\varphi\left(\rho_{r}, \rho_{r+1}\right) \varphi\left(\rho^{*}, \mathcal{V} \rho^{*}\right)}{1+\varphi\left(\rho_{r}, \rho^{*}\right)}\right] .
\end{aligned}
$$

Taking the limit as $r \rightarrow \infty$ and using (2.5), we get a contradiction to $\varphi\left(\rho^{*}, \mathcal{V} \rho^{*}\right)>0$. Thus $\varphi\left(\rho^{*}, \mathcal{V} \rho^{*}\right)=0$. This yields that $\rho^{*}=\mathcal{V} \rho^{*}$. Hence it is proved.

Example 2.2. Let $\mathcal{Q}=\{0,1,2\}$. Define $\sigma: \mathcal{Q} \times \mathcal{Q} \rightarrow[1, \infty)$ and $\varphi: \mathcal{Q} \times \mathcal{Q} \rightarrow[1, \infty)$ as $\sigma(\rho, \kappa)=1+\rho \kappa$ and

$$
\begin{aligned}
& \varphi(2,2)=\varphi(0,0)=\varphi(1,1)=0, \\
& \varphi(2,0)=\varphi(0,2)=5, \quad \varphi(0,1)=\varphi(0,1)=10, \\
& \varphi(1,2)=\varphi(2,1)=30 .
\end{aligned}
$$

Now, define

$$
\mathcal{V}: Q \rightarrow Q
$$

by

$$
V \rho= \begin{cases}0, & \text { if } \rho \in\{0,2\} \\ 2, & \text { if } \rho=1\end{cases}
$$

and choose $\alpha=\frac{1}{2}$ and $\beta=\frac{1}{3}$.

Case 01: If $\rho=0, k=1$, we have

$$
\varphi(\mathcal{V} 0, \mathcal{V} 1)=\varphi(0,2)=5=\frac{1}{2}(10)=\frac{1}{2} \varphi(0,1)+\frac{1}{3} \frac{\varphi(0,0) \varphi(1,2)}{1+\varphi(0,1)} .
$$

Case 02: If $\rho=0, k=2$, we have

$$
\varphi(\mathcal{V} 0, \mathcal{V} 2)=\varphi(0,0)=0<\frac{1}{2}(5)=\frac{1}{2} \varphi(0,2)+\frac{1}{3} \frac{\varphi(0,0) \varphi(2,0)}{1+\varphi(0,2)} .
$$

Case 03: If $\rho=1, k=2$, we have

$$
\left.\varphi(\mathcal{V} 1, \mathcal{V} 2)=\varphi(2,0)=5<15+\frac{50}{31}=\frac{1}{2} \varphi(1,2)+\frac{1}{3} \frac{\varphi(1,2) \varphi(2,0)}{1+\varphi(1,2)}\right) .
$$

Case 04: If $\rho=\kappa=0, \rho=\kappa=1, \rho=\kappa=2$, we have

$$
\varphi\left(\mathcal{V}_{\rho}, \mathcal{V}_{\kappa}\right)=0 .
$$

Consequently,

$$
\varphi\left(\mathcal{V} \rho, \mathcal{V}_{\kappa}\right) \leqslant \alpha(\varphi(\rho, \mathcal{V} \rho)+\varphi(\kappa, \mathcal{V} \kappa)),
$$

$\forall \rho, \kappa \in Q$. Thus the assertions of above result are fulfilled and $\mathcal{V}$ has a unique fixed point, that is, $\rho=0$.

\section{Special Cases:}

Corollary 2.3. Let $\left(Q, \varphi_{e}\right)$ be a complete EbMS. Let $\mathcal{V}: Q \rightarrow Q$ be such that

$$
\varphi_{e}\left(\mathcal{V} \rho, \mathcal{V}_{\kappa}\right) \leqslant \alpha \varphi_{e}(\rho, \kappa)+\beta \frac{\varphi_{e}(\rho, \mathcal{V} \rho) \varphi_{e}\left(\kappa, V_{\kappa}\right)}{1+\varphi_{e}(\rho, \kappa)},
$$

$\forall \rho, \kappa \in Q$, where $\alpha, \beta \in[0,1)$ such that $\lambda=\alpha+\beta<1$. For $\rho_{0} \in \mathcal{Q}$, take $\rho_{\mathrm{r}}=\mathcal{V}^{\mathrm{r}} \rho_{0}$. Suppose that 


$$
\sup _{m \geqslant 1} \lim _{i \rightarrow \infty} \frac{\sigma\left(\rho_{i+1}, \rho_{i+2}\right) \sigma\left(\rho_{i+1}, \rho_{m}\right)}{\sigma\left(\rho_{i}, \rho_{i+1}\right)}<\frac{1}{\lambda} .
$$

Also, assume that $\forall \rho \in \mathcal{Q}$, we have $\lim _{r \rightarrow \infty} \sigma\left(\rho_{\mathrm{r}}, \rho\right)$ and $\lim _{\mathrm{r} \rightarrow \infty} \sigma\left(\rho, \rho_{\mathrm{r}}\right)$ exist and are finite. Then, $\exists \rho^{*} \in \mathcal{Q}$ such that $\mathcal{V} \rho^{*}=\rho^{*}$.

Proof. Take $\sigma(\rho, \omega)=\sigma(\omega, \mathrm{k})$ in Theorem 2.1.

Corollary 2.4. Let $\left(\mathcal{Q}, \varphi_{\mathrm{b}}\right)$ be a complete $b M S$ and let $\mathcal{V}: \mathcal{Q} \rightarrow \mathcal{Q}$. If $\exists \alpha, \beta \in[0,1)$ such that $\lambda=\alpha+\beta<1$ and

$$
\varphi_{\mathrm{b}}\left(\mathcal{V}_{\rho}, \mathcal{V}_{\kappa}\right) \leqslant \alpha \varphi_{\mathrm{b}}(\rho, \kappa)+\beta \frac{\varphi_{\mathrm{b}}(\rho, \mathcal{V} \rho) \varphi_{\mathrm{b}}\left(\kappa, \mathcal{V}_{\kappa}\right)}{1+\varphi_{\mathrm{b}}(\rho, \kappa)}, \quad \forall \rho, \kappa \in \mathcal{Q},
$$

then $\exists \rho^{*} \in \mathcal{Q}$ such that $\mathcal{V} \rho^{*}=\rho^{*}$.

Proof. Take $\sigma(\rho, \omega)=\sigma(\omega, \kappa)=s \geqslant 1$ in Theorem 2.1.

Corollary 2.5. Let $(Q, \varphi)$ be a complete $M S$ and let $\mathcal{V}: Q \rightarrow Q$. If $\exists \alpha, \beta \in[0,1)$ such that $\lambda=\alpha+\beta<1$ and

$$
\varphi\left(\mathcal{V} \rho, \mathcal{V}_{\kappa}\right) \leqslant \alpha \varphi(\rho, \kappa)+\beta \frac{\varphi(\rho, \mathcal{V} \rho) \varphi\left(\kappa, \mathcal{V}_{\kappa}\right)}{1+\varphi(\rho, \kappa)}, \quad \forall \rho, \kappa \in \mathcal{Q},
$$

then $\exists \rho^{*} \in \mathcal{Q}$ such that $\mathcal{V} \rho^{*}=\rho^{*}$.

Proof. Take $\sigma(\rho, \omega)=\sigma(\omega, \mathrm{k})=1$ in Theorem 2.1.

\section{References}

[1] T. Abdeljawad, N. Mlaiki, H. Aydi, N. Souayah, Double Controlled Metric Type Spaces and Some Fixed Point Results, Mathematics, 6 (2018), 10 pages. 1

[2] J. Ahmad, A. E. Al-Mazrooei, Y. J. Cho, Y.-O. Yang, Fixed point results for generalized $\Theta$-contractions, J. Nonlinear Sci. Appl., 10 (2017), 2350-2358.

[3] J. Ahmad, A. S. Al-Rawashdeh, A. Azam, Fixed point results for $\{\alpha, \xi\}$-expansive locally contractive mappings, J. Inequal. Appl., 2014 (2014), 10 pages.

[4] J. Ahmad, A. Al-Rawashdeh, A. Azam, New fixed point theorems for generalized F-contractions in complete metric spaces, Fixed Point Theory Appl., 2015 (2015), 18 pages.

[5] L. A. Alnaser, D. Lateef, H. A. Fouad, J. Ahmad, Relation theoretic contraction results in F-metric spaces, J. Nonlinear Sci. Appl., 12 (2019), 337-344.

[6] B. Alqahtani, E. Karapinar, A. Öztürk, On $(\alpha-\psi)-\mathrm{K}-$ contractions in the extended b-metric space, Filomat, 32 (2018), 5337-5345.

[7] Z. Aslam, J. Ahmad, N. Sultana, New common fixed point theorems for cyclic compatible contractions, J. Math. Anal., 8 (2017), 1-12.

[8] A. Azam, N. Mehmood, J. Ahmad, S. Radenović, Multivalued fixed point theorems in cone b-metric spaces, J. Inequal. Appl., 2013 (2013), 9 pages.

[9] S. Banach, Sur les opérations dans les ensembles abstraits et leur application aux équations intégrales, Fund. Math., 3 (1922), 133-181. 1

[10] S. Czerwik, Contraction mappings in b-metric spaces, Acta Math. Inform. Univ. Ostraviensis, 1 (1993), 5-11. 1, 1.1

[11] B. Fisher, Mappings satisfying a rational inequality, Bull. Math. Soc. Sci. Math. R. S. Roumanie (N.S.), 24 (1980), 247-251. 1, 1

[12] H. P. Huang, S. Radenović, Some fixed point results of generalised Lipschitz mappings on cone b-metric spaces over Banach algebras, J. Comput. Anal. Appl., 20 (2016), 566-583.

[13] N. Hussain, J. Ahmad, A. Azam, On Suzuki-Wardowski type fixed point theorems, J. Nonlinear Sci. Appl., 8 (2015), 1095-1111.

[14] N. Hussain, A. E. Al-Mazrooei, J. Ahmad, Fixed point results for generalized $(\alpha-\eta)-\Theta$ contractions with applications, J. Nonlinear Sci. Appl., 10 (2017), 4197-4208.

[15] N. Hussain, C. Vetro, F. Vetro, Fixed point results for $\alpha$-implicit contractions with application to integral equations, Nonlinear Anal. Model. Control, 21 (2016), 362-378.

[16] T. Kamran, M. Samreen, Q. UL Ain, A generalization of b-metric space and some fixed point theorems, Mathematics, 5 (2017), 7 pages. 1

[17] R. Kannan, Some results on fixed points, Bull. Calcutta Math. Soc., 60 (1968), 71-76. 
[18] E. Karapınar, P. Kumari, D. Lateef, A New Approach to the Solution of the Fredholm Integral Equation via a Fixed Point on Extended b-Metric Spaces, Symmetry, 10 (2018), 13 pages.

[19] M. A. Kutbi, J. Ahmad, A. Azam, On fixed points of $\alpha-\psi$-contractive multi-valued mappings in cone metric spaces, Abst. Appl. Anal., 2013 (2013), 13 pages.

[20] D. Lateef, J. Ahmad, Dass and Gupta's fixed point theorem in F-metric spaces, J. Nonlinear Sci. Appl., 12 (2019), 405-411.

[21] N. Mlaiki, H. Aydi, N. Souayah, T. Abdeljawad, Controlled Metric Type Spaces and the Related Contraction Principle, Mathematics, 6 (2018), 7 pages. 1, 1.3, 1.4, 1.5

[22] W. Onsod, T. Saleewong, J. Ahmad, A. E. Al-Mazrooei, P. Kumam, Fixed points of a $\Theta$-contraction on metric spaces with a graph, Commun. Nonlinear Anal., 2 (2016), 139-149.

[23] D. Wardowski, Fixed points of a new type of contractive mappings in complete metric spaces, Fixed Point Theory Appl., 2012 (2012), 6 pages. 1 